\title{
Alkaline phosphatase flare with hyperostosis of bone metastases in lung adenocarcinoma treated with gefitinib
}

\author{
Hiroto Kaneko', Kazuho Shimura', Yosuke Matsumoto', Mihoko Yoshida', Masafumi Taniwaki', Junya Kuroda ${ }^{2}$ \\ ${ }^{1}$ Department of Hematology, Aiseikai Yamashina Hospital, 19-4 Shichouno-cho, Yamashina-ku, Kyoto 607-8086, Japan. \\ ${ }^{2}$ Department of Hematology, Kyoto Prefectural University of Medicine, 465 Kajii-cho, Kawaramachi Hirokoji, Kamigyou-ku, Kyoto 602-8566, Japan.
}

Correspondence to: Dr. Hiroto Kaneko, Department of Hematology, Aiseikai Yamashina Hospital, 19-4 Shichouno-cho, Yamashina-ku, Kyoto 607-8086, Japan. E-mail: hirotok@koto.kpu-m.ac.jp

How to cite this article: Kaneko H, Shimura K, Matsumoto Y, Yoshida M, Taniwaki M, Kuroda J. Alkaline phosphatase flare with hyperostosis of bone metastases in lung adenocarcinoma treated with gefitinib. J Cancer Metastasis Treat 2017;3:34-7.

Article history:

Received: 19-12-2016

Accepted: 12-01-2017

Published: 23-02-2017

Key words:

Alkaline phosphatase,

bone metastasis,

hyperostosis,

gefitinib

\section{ABSTRACT}

\begin{abstract}
Alkaline phosphatase (ALP) flare has been reported to occur during cancer treatment as a favorable event, particularly in the presence of bone metastasis. There have been only a few reports in lung cancer and associated radiographic findings have seldom been described. The authors observed ALP flare in a female patient with lung adenocarcinoma soon after the initiation of gefitinib. Moreover, on computed tomography, metastatic lesions of the rib and thoracic spine showed marked hyperostosis, with sizes larger than the original bone structure, suggesting efficacy of gefitinib. The significance of such hyperostosis should be elucidated.
\end{abstract}

\section{INTRODUCTION}

Alkaline phosphatase (ALP) flare is known as a transient elevation of serum ALP value in cancer patients with bone metastasis, particularly in breast or prostatic cancer. ${ }^{[1]}$ It is generally accepted that ALP flare emerges when systemic treatment is effective, since osteosclerotic change of the osteolytic lesion is seen radiographically. ${ }^{[2,3]}$ Although osteosclerosis without ALP flare has been well documented, ${ }^{[4,5]}$ ALP flare is reported to occur in only $5 \%$ of non-small cell lung cancer (NSCLC) patients treated with epidermal growth factor tyrosine kinase inhibitor (EGFR-TKI). ${ }^{[6]}$ Since the frequency is relatively low, precise characteristics of ALP flare in NSCLC have not yet been elucidated, but it seems important not to misinterpret ALP flare as a progression of bone lesion. ${ }^{[4]}$ We report a patient who presented ALP flare and unusual hyperostosis of metastatic bone lesions shortly after initiation of EGFRTKI for lung adenocarcinoma.

\section{CASE REPORT}

A 66-year-old woman visited our department

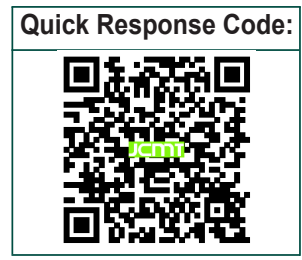


complaining of backache that had developed two weeks before. She had no history of smoking. Physical examination revealed no significant findings. However, chest X-ray showed a mass shadow at the left upper lung field, and computed tomography (CT) confirmed a tumor of $4.5 \mathrm{~cm}$ in diameter with irregular margins at the left upper lobe. The tumor was accompanied by ground glass-like consolidation [Figure 1A]. Osteolytic lesions of the fourth thoracic spine [Figure 2A] and the fourth left rib [Figure 2B] were also shown. Laboratory data demonstrated elevation of serum carcinoembryonic antigen (CEA) [Table 1]. ALP was also elevated at $379 \mathrm{IU} / \mathrm{L}$ (normal range from 104 to 338). CT-guided needle biopsy was carried out and the acquired specimen was pathologically diagnosed as adenocarcinoma. The tumor was also found to carry a mutation of EGFR (L858R). Treatment with gefitinib began and her subjective symptom was relieved quickly within several days.

On treatment day 13, ALP increased to $952 \mathrm{IU} / \mathrm{L}$, about 2.5 times the pretreatment level. Serum transaminases were also simultaneously elevated (AST 101 and ALT 168), suggesting gefitinib-induced liver dysfunction. Serum Ca remained within normal limits. Electrophoretic analysis showed that ALP-isozyme 1 accounted for $8.6 \%$, ALP-2 56.3\%, and ALP-3 35.1\%, respectively. Although bone-derived ALP-3 was seen to increase, ALP-2 liver-derived isozyme had the larger increase, probably because of simultaneous druginduced liver injury.

Since the significance of elevated ALP was unclear, another CT was carried out on treatment day 38 . Results showed the primary pulmonary tumor was reduced to $3 \mathrm{~cm}$ in diameter [Figure 1B]. Previously osteolytic lesions had become osteosclerotic. Lesion sizes are evidenced excessive growth, larger than the size of the original bones [Figure $2 \mathrm{C}$ and $\mathrm{D}$ ]. ALP gradually decreased and liver dysfunction regressed [Figure 3]. CEA also decreased to $34.7 \mathrm{ng} / \mathrm{mL}$, about one fourth of the maximum value [Figure 3].

\section{DISCUSSION}

A phenomenon, so called osteoblastic flare, has originally been recognized as a transient increased uptake of radiotracer of bone scintigraphy. ${ }^{[1,7]}$ However, ALP appeared to replace later because of infrequent use of bone scan, rapid and easy application of ALP, and coincident fluctuation of both. ${ }^{[6]}$ Our patient demonstrated a rapid improvement of bone pain and tumor regression by gefitinib. This study might support the previous report by Arai et al. ${ }^{[3]}$ suggesting a favorable response to EGFR-TKI in case of ALP flare. It is of interest that Shimazaki et al. ${ }^{\left[{ }^{[8]}\right.}$ first observed ALP flare-like phenomenon in a patient with multiple myeloma who received bortezomib for recurrent massive bone lesions. Their patient showed a transient ALP-3 increase without disease progression. Recent extreme efficacy of novel therapeutic agents might

\section{Table 1: Laboratory data on admission}

\begin{tabular}{ll}
\hline Inspection item & Value \\
\hline White blood cells & $10,790 \mu \mathrm{L}$ \\
Red blood cells & $456 \times 10^{4} \mu \mathrm{L}$ \\
Hemoglobin & $13.6 \mathrm{~g} / \mathrm{dL}$ \\
Hematocrit & $40.5 \%$ \\
Platelets & $38.5 \times 10^{4} \mu \mathrm{L}$ \\
Carcinoembryonic entigen & $137.9 \mathrm{ng} / \mathrm{mL}(0-5.0)^{*}$ \\
Sialyl Lewis-X antigen & $110 \mathrm{ng} / \mathrm{mL}(<38.0)^{*}$ \\
Alkaline phosphatase & $\mathbf{3 7 9} \mathrm{IU} / \mathrm{L}(104-338)^{*}$ \\
Lactate dehydrogenase & $468 \mathrm{IU} / \mathrm{L}(108-221)^{*}$ \\
Asparate aminotransferase & $25 \mathrm{IU} / \mathrm{L}$ \\
Alanine aminotranferase & $14 \mathrm{IU} / \mathrm{L}$ \\
Total bilirubin & $0.7 \mathrm{mg} / \mathrm{dL}$ \\
Leucine aminopeptidase & $59 \mathrm{IU} / \mathrm{L}$ \\
Gamma glutamyl transpeptidase & $37 \mathrm{IU} / \mathrm{L}$ \\
Albumin & $3.9 \mathrm{~g} / \mathrm{dL}$ \\
Blood urea nitrogen & $11.5 \mathrm{mg} / \mathrm{dL}$ \\
Creatinine & $0.76 \mathrm{mg} / \mathrm{dL}$ \\
Uric acid & $4.8 \mathrm{mg} / \mathrm{dL}$ \\
Calcium & $10.2 \mathrm{mg} / \mathrm{dL}$ \\
Natrium & $142 \mathrm{mEq} / \mathrm{L}$ \\
Kalium & $4.2 \mathrm{mEq} / \mathrm{L}$ \\
Chloride & $103 \mathrm{mEq} / \mathrm{L}$ \\
C-reactive protein & $0.10 \mathrm{mg} / \mathrm{dL}$ \\
HBsAg & negative \\
HBsAb & negative \\
HBcAb & negative \\
HCVAb & negative \\
\hline Abnorma data are in bold &
\end{tabular}

*Abnormal data are in bold. Their normal ranges are indicated in the following parentheses
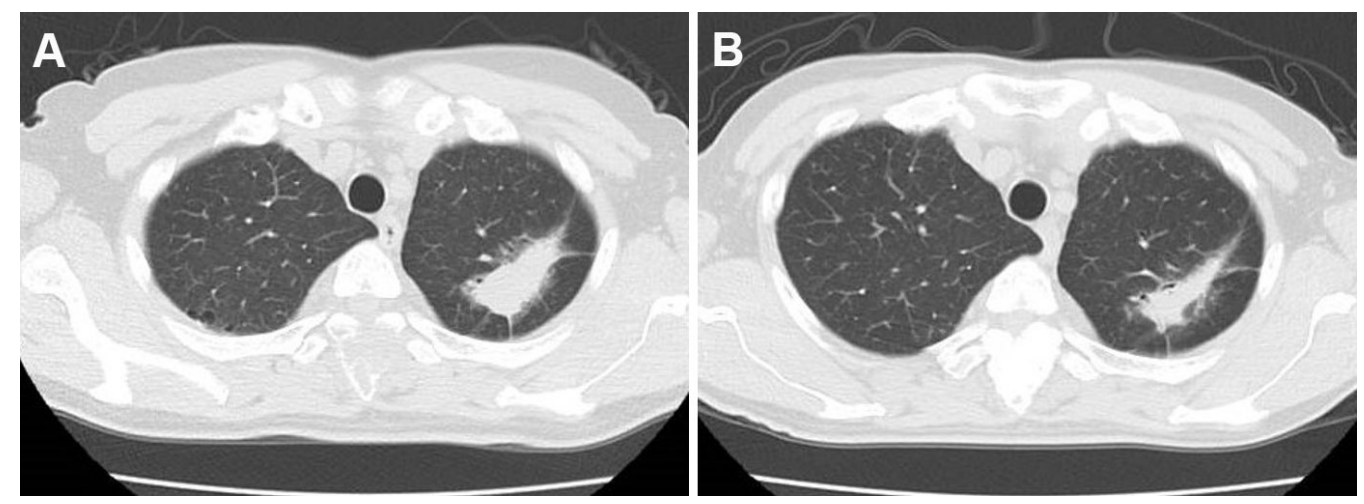

Figure 1: (A) Irregular shaped tumor at left upper lobe; (B) the tumor reduced in size after the initiation of gefitinib 

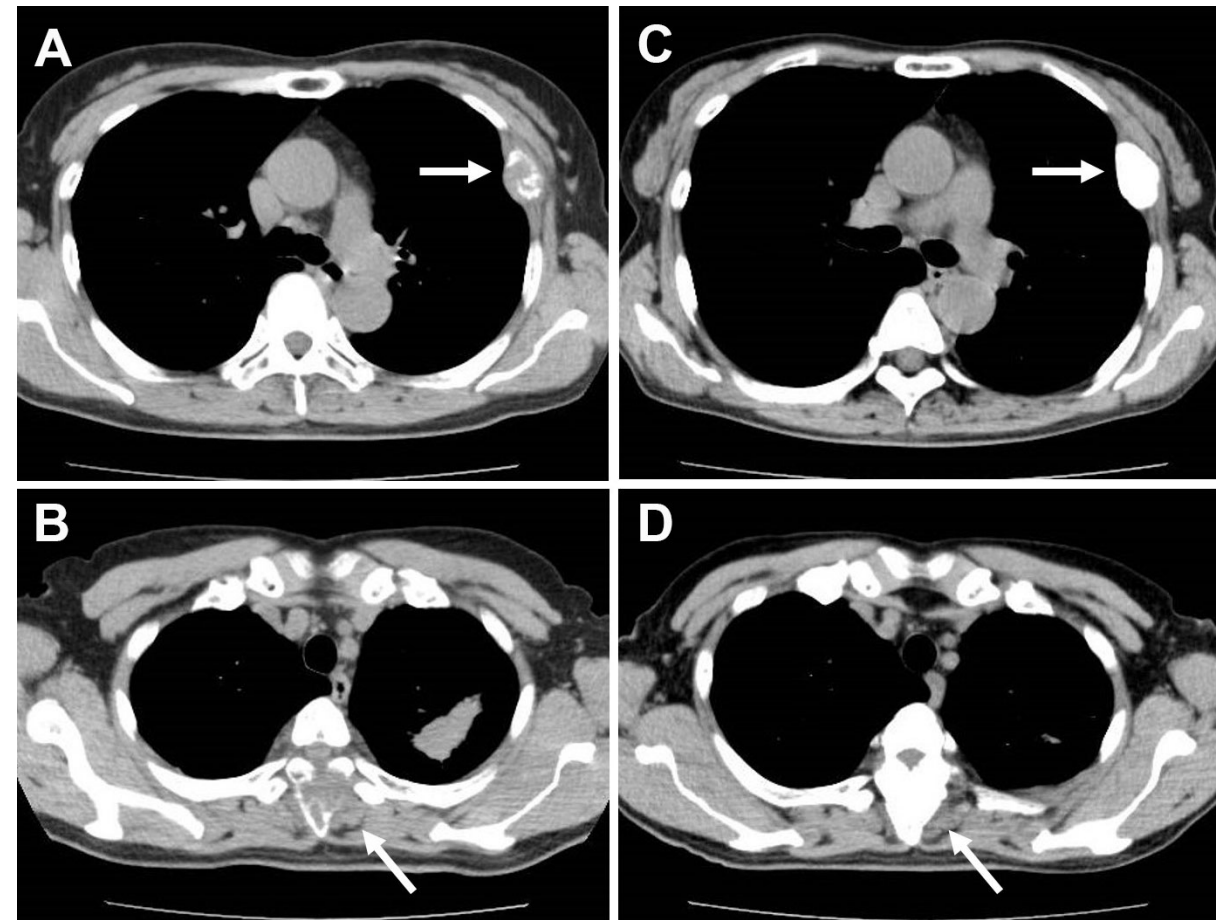

Figure 2: Osteolytic metastatic lesions of the left fourth rib $(A)$ and the fourth thoracic spine $(B)$; hyperostosis of the lesions was seen by gefitinib treatment $(C, D)$

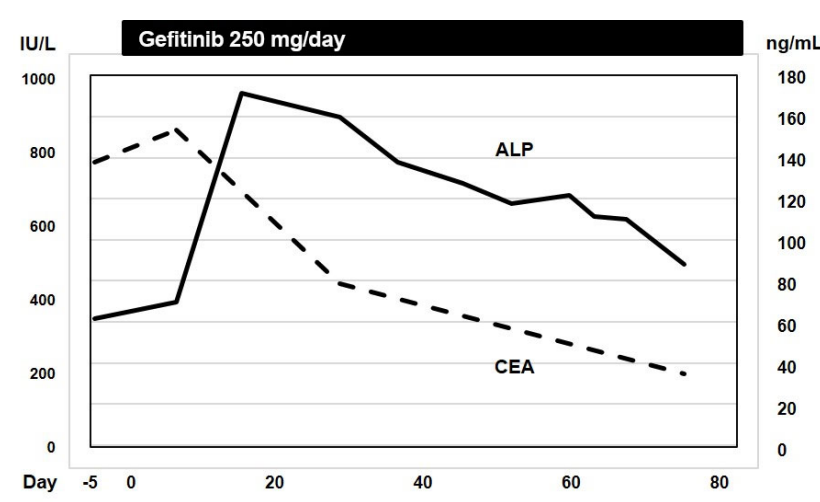

Figure 3: Transient ALP flare after the initiation of gefitinib is shown. CEA continued to decrease. ALP: alkaline phosphatase; CEA: carcinoembryonic antigen

induce ALP flare in various malignancies with bone lesions. It is also intriguing that NSCLC patients even without definite bone metastasis who experienced ALP flare showed better survival. ${ }^{[6]}$ Bone-derived ALP flare that might represent osteoblastic reaction is supposed to predict a response to EGFR-TKI, although a precise mechanism is to be elucidated. A previous investigation referred to the reduction of osteoclast differentiation in the bone marrow caused by gefitinib, ${ }^{[9]}$ suggesting a relation between therapeutic response and osteoblastic reaction. It is notable that ALP flare has mainly been found in Japanese patients. There might be racial differences among biological responses to EGFR-TKI.

To our knowledge, CT findings of improvement of bone metastatic lesions accompanied by ALP flare have seldom been demonstrated previously. Although osteosclerotic changes within the metastatic lesions were depicted in these studies, ${ }^{[4,5]}$ our patient showed excessive calcification including metastatic lesions as shown in Figure 2. There has been no similar description of such hyperostosis in English literature. We hypothesized that radiographically latent tumor tissue around the bone metastasis might also be calcified by EGFR-TKI therapy, resulting in hyperostosis. On the other hand, radiographic osteoblastic change of metastasis before treatment was also regarded as a favorable prognostic marker for NSCLC treated with EGFR-TKI. ${ }^{[10]}$ It is suggested that osteoblastic reaction regardless before or after the initiation of treatment might influence tumor reduction, as well as ALP flare phenomenon.

Moreover, drug-induced liver injury might cover ALP flare if liver transaminases or biliary tract markers also markedly elevate as well as ALP. Drug-induced liver damage was reported to be seen in 5 of $41(12.5 \%)$ patients with NSCLC who were treated with EGFRTKI. ${ }^{[11]}$ Thus, the frequency of ALP flare is supposed to be much higher than practically observed. Negativity of hepatitis viral markers or outstandingly elevated ALP among liver function markers might help detecting ALP flare. Physicians should pay more attention to ALP flare as well as therapeutic response of radiographic findings of bone metastasis to elucidate the significance of osteoblastic reactions in the outcome of lung cancer. At least, ALP flare strongly suggests that EGFR-TKI 
should not be discontinued even if adverse effects of other organs might emerge.

\section{Authors' contributions}

Patient's treatment and manuscript's preparation: $\mathrm{H}$. Kaneko

Data acquisition: M. Yoshida

Study design: K. Shimura, Y. Matsumoto

Manuscript's review: M. Taniwaki, J. Kuroda

\section{Financial support and sponsorship}

None.

\section{Conflicts of interest}

There are no conflicts of interest.

\section{Patient consent}

All involved patients give their consent forms.

\section{Ethics approval}

Ethics approval was obtained from Institute Review Board for case reports.

\section{REFERENCES}

1. Coleman RE, Whitaker KB, Moss DW, Mashiter G, Fogelman I, Rubens RD. Biochemical prediction of response of bone metastases to treatment. Br J Cancer 1988;58:205-10.

2. Arai Y, Kojima A. A case of lung cancer with alkaline phosphatase flare phenomenon during gefitinib therapy. Nihon Kokyuki Gakkai Zasshi 2007;45:962-6. (in Japanese)

3. Hashisako M, Wakamatsu K, Ikegami S, Kumazoe H, Nagata
$\mathrm{N}$, Kajiki A. Flare phenomenon following gefitinib treatment of lung adenocarcinoma with bone metastasis. Tohoku J Exp Med 2012;228:163-8

4. Garfield D. Increasing osteoblastic lesions as a manifestation of a major response to gefitinib. $J$ Thorac Oncol 2006;1:859-60.

5. Lind JS, Postmus PE, Smit EF. Osteoblastic bone lesions developing during treatment with erlotinib indicate major response in patients with non-small cell lung cancer: a brief report. J Thorac Oncol 2010;5:554-7.

6. Yasuda Y, Kawamura K, Ichikado K, Yoshioka M. Alkaline phosphatase flare phenomenon following epidermal growth factortyrosine kinase inhibitor treatment of non-small cell lung cancer: report of a case and case review. Respir Med Case Rep 2014;13:51-3.

7. Chao HS, Chang CP, Chiu CH, Chu LS, Chen YM, Tsai CM. Bone scan flare phenomenon in non-small-cell lung cancer patients treated with gefitinib. Clin Nucl Med 2009;34:346-9.

8. Shimazaki C, Uchida R, Nakano S, Namura K, Fuchida S, Okano A, Okamoto M, Inaba T. High serum bone-specific alkaline phosphatase level after bortezomib-combined therapy in refractory multiple myeloma: possible role of bortezomib on osteoblast differentiation. Leukemia 2005;19:1102-3.

9. Normanno N, De Luca A, Aldinucci D, Maiello MR, Mancino M, D'Antonio A, De Fillippi R, Pinto A. Gefitinib inhibits the ability of human bone marrow stromal cells to induce osteoclast differentiation: implications for the pathogenesis and treatment of bone metastasis. Endocr Relat Cancer 2005;12:471-82.

10. Pluquet E, Cadranel J, Legendre A, Beau Faller M, Souquet PJ, Zalcman G, Perol M, Fraboulet G, Oliveiro G, De Fraipont F, Quoix E, Lantuejoul S, Milleron B, Moro-Sibilot D. Osteoblastic reaction in non-small cell lung carcinoma and its association to epidermal growth factor receptor tyrosine kinase inhibitors response and prolonged survival. J Thorac Oncol 2010;5:491-6.

11. Yoshimoto A, Kasahara K, Kimura H, Kita T, Fujimura M, Nakao S. Transient liver injury caused by gefitinib. Nihon Kokyuki Gakkai Zasshi 2004;42:56-61. (in Japanese) 